\title{
Two-Dimensional Electronic Spectroscopy of a Minimal Photosystem I Complex Reveals the Rate of Primary Charge Separation
}

Parveen Akhtar, Ido Caspy, Paweł J. Nowakowski, Tirupathi Malavath, Nathan Nelson, Howe-Siang Tan, Petar H. Lambrev

\section{SUPPLEMENTARY FigURES}

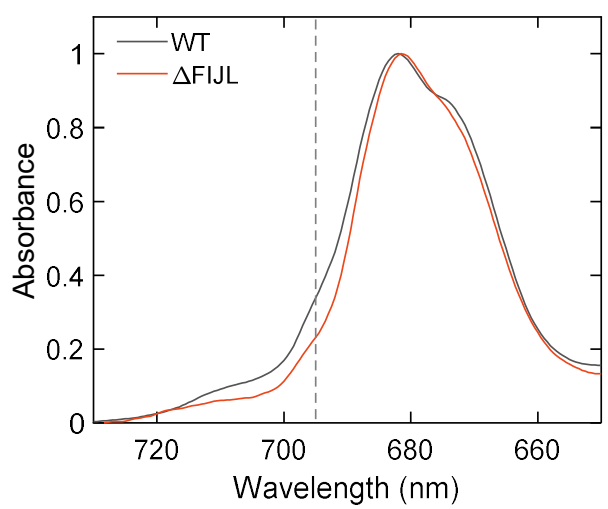

Figure S 1. Comparison of the $77 \mathrm{~K}$ absorption spectra of trimeric Photosystem I from Synechocystis PCC 6803 (WT) and the minimal Photosystem I lacking subunits PsaF, PsaI, PsaJ, PsaL ( $\Delta$ FIJL). The dashed vertical line is at $695 \mathrm{~nm}$.

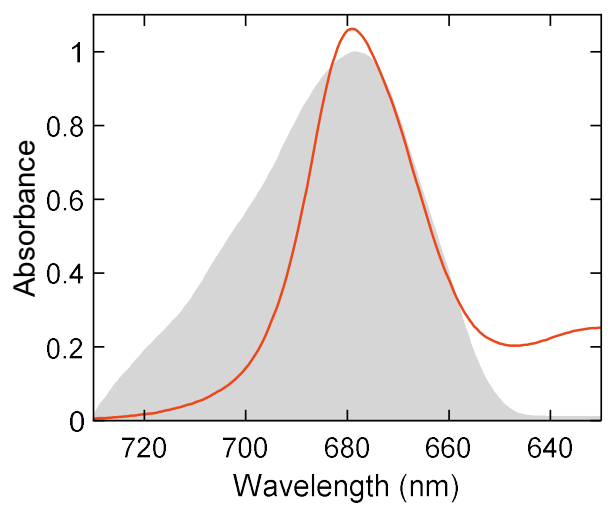

Figure S 2. Excitation pulse spectrum used in 2DES (grey shaded) overlaid with the room-temperature absorption spectrum of the minimal Photosystem I from Synechocystis $\Delta$ FIJL. 
a

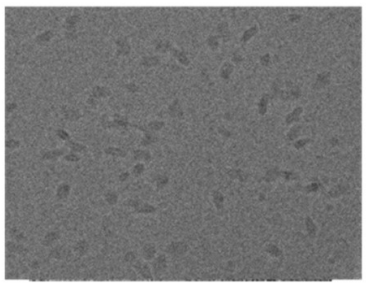

C

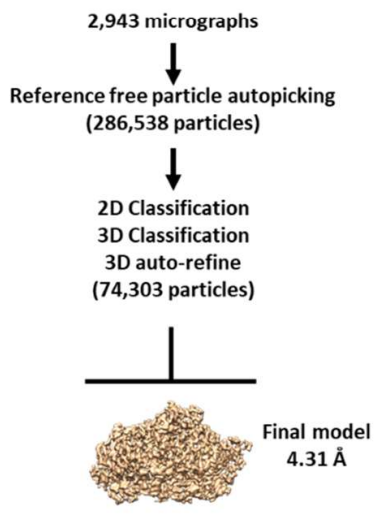

e

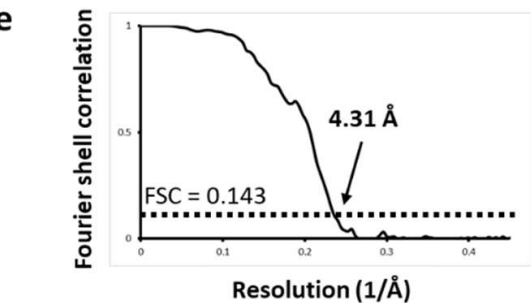

b

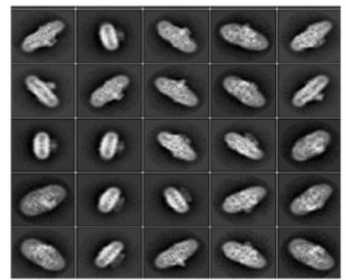

d

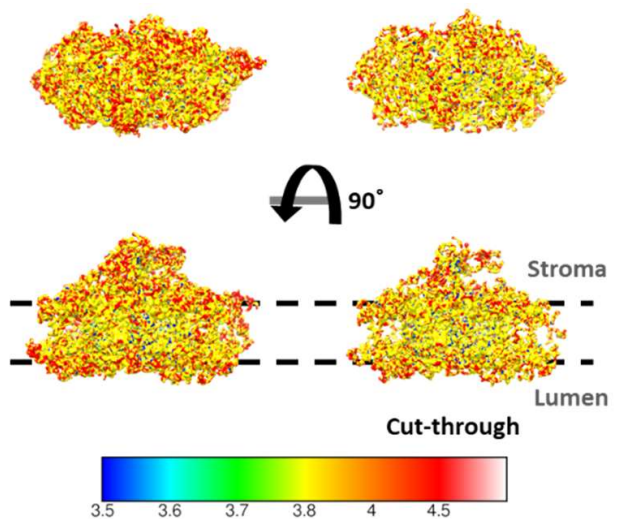

Figure S 3. $\Delta$ FIJL Photosystem I cryo-EM analysis. a) A zoomed-in view of a $\Delta$ FIJL micrograph. b) Representing 2D classes. c) Cryo-EM data processing workflow. d) Local resolution of the complete $\Delta$ FIJL (left) and map cutthrough (right). e) RELION Fourier Shell Correlation postprocessing result.
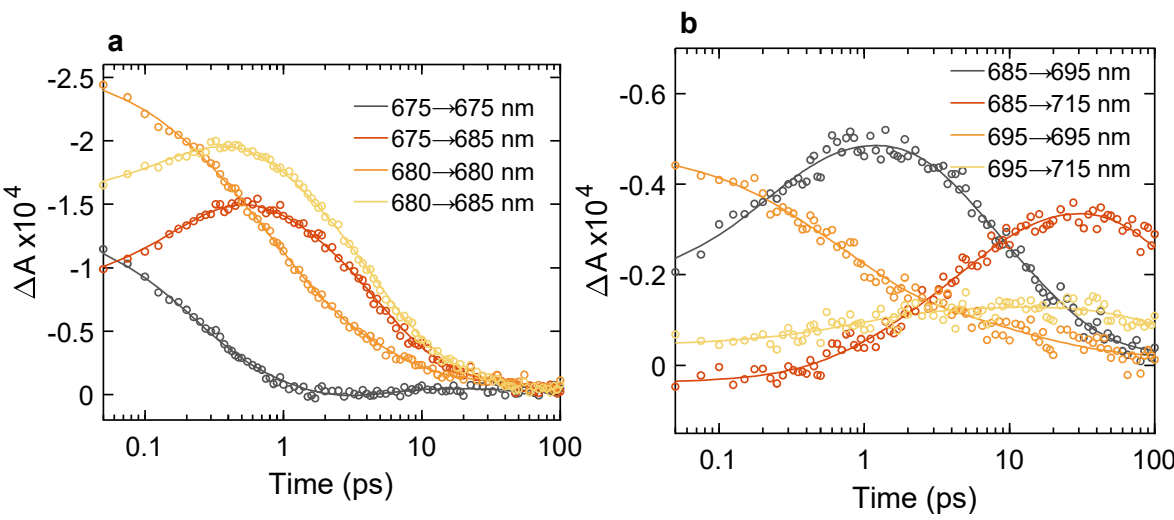

Figure S 4. Kinetic traces at selected wavelengths $\lambda_{\tau} \rightarrow \lambda_{\mathrm{t}}$. The symbols represent the measured data points and the lines are obtained by global multiexponential fitting. These traces highlight two main points 1) fast relaxation upon excitation at blue edge of the $\mathrm{Q}_{\mathrm{y}}$ absorption band - bulk antenna excitation; 2) absence of red 
states upon excitation at the red edge of $\mathrm{Q}_{\mathrm{y}}$ absorption $695 \mathrm{~nm}(695 \rightarrow 715 \mathrm{~nm}$ trace). This indicates that the red states are populated by energy transfer from shorter-wavelength antenna Chls only.
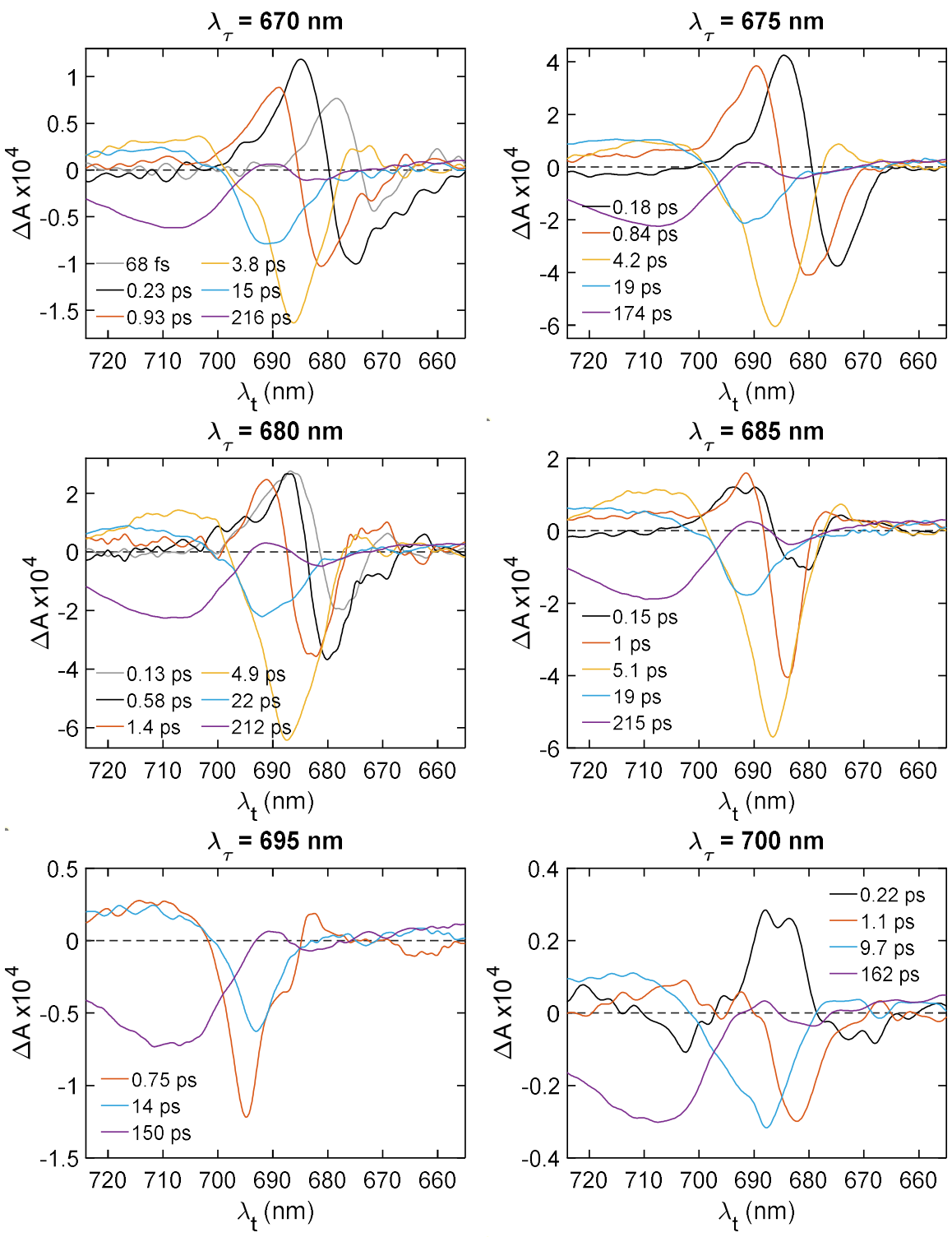

Figure S 5. Decay-associated absorption difference spectra obtained from the $77 \mathrm{~K}$ 2DES data analysed separately for excitation wavelengths $670,675,680,685,695$ and $700 \mathrm{~nm}$. 

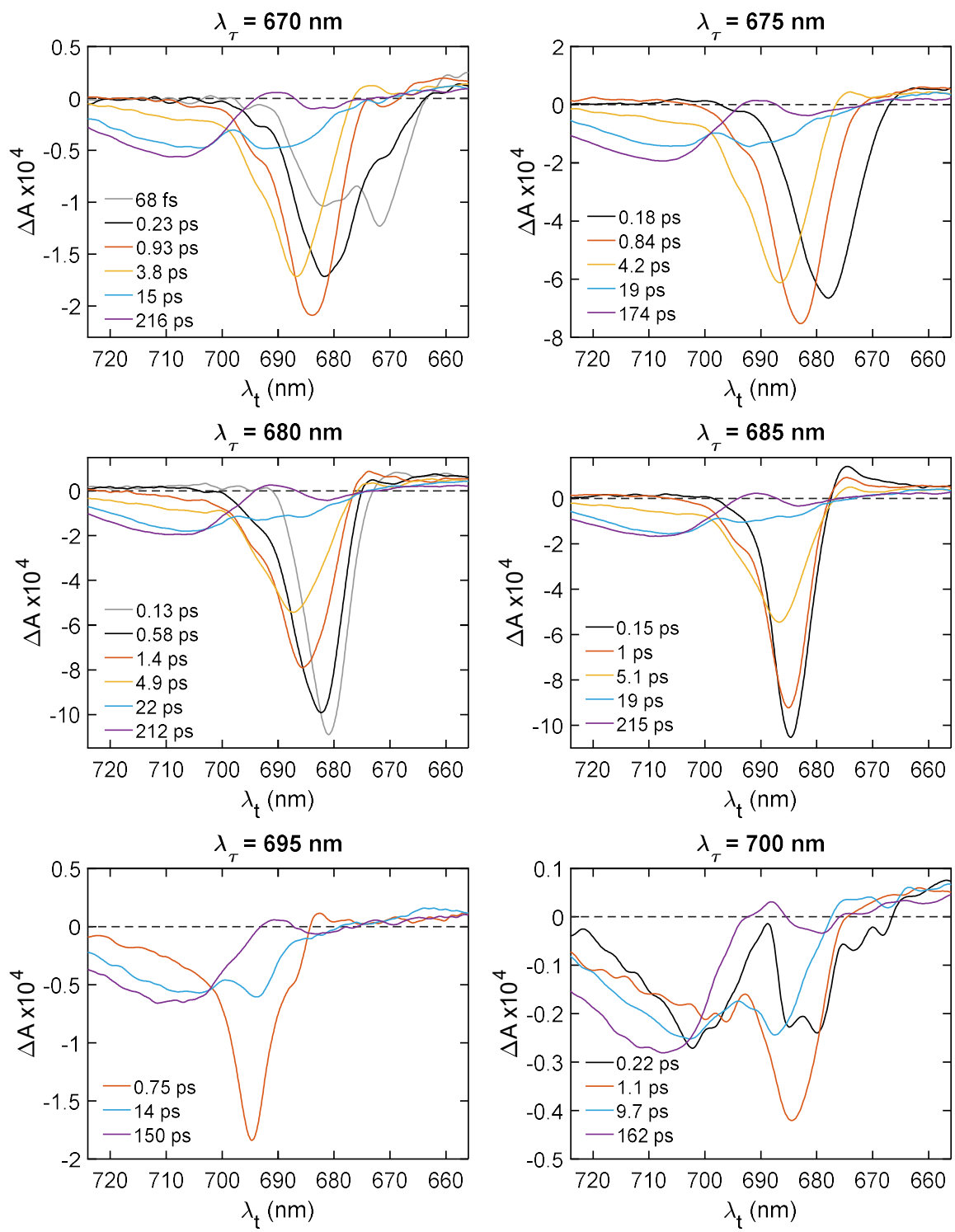

Figure S 6. Evolution-associated absorption difference spectra (EADS) obtained from analyzing slices of the 2DES data at selected excitation wavelengths $\lambda_{\tau}$ with a sequential unbranched kinetic model $A \rightarrow B \rightarrow C \rightarrow \ldots$ where subsequent steps are progressively slower. The corresponding decay lifetimes (inverse rate constants) are indicated. 

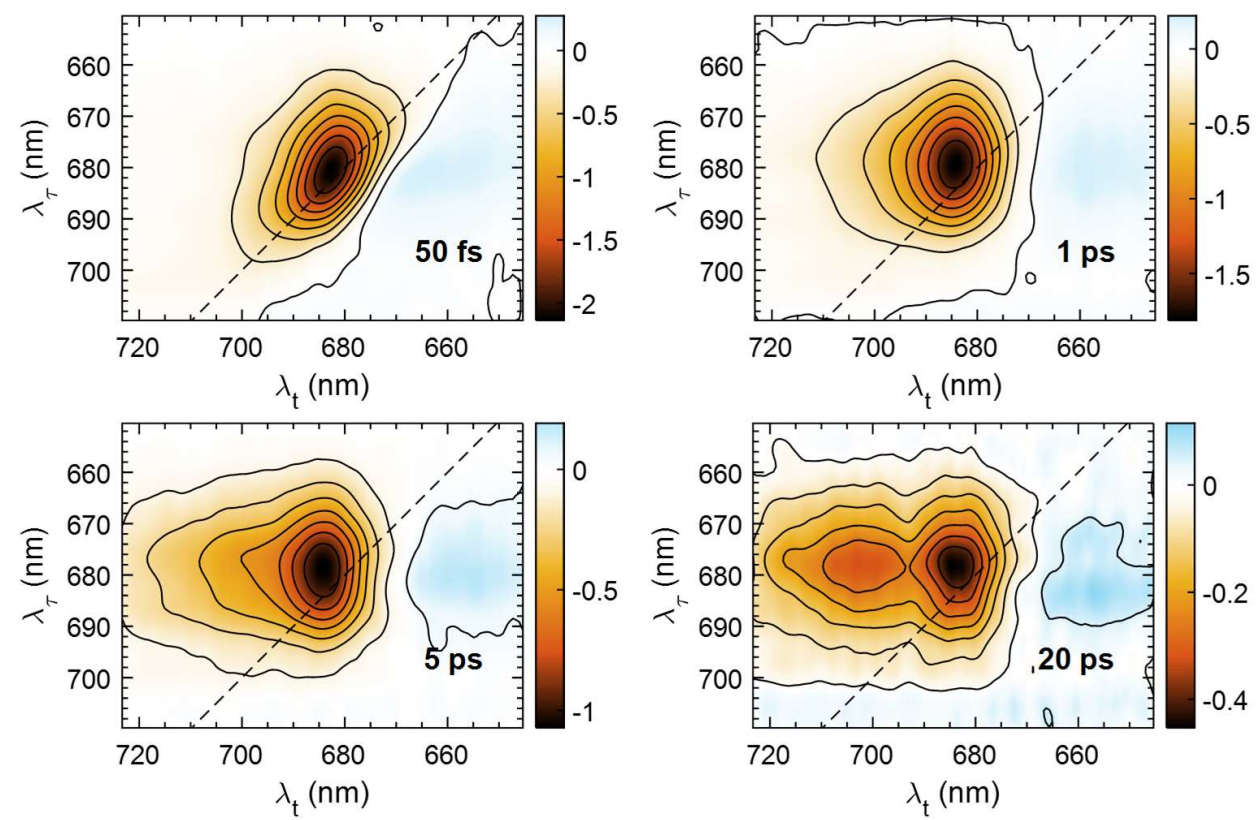

Figure S 7. Selected purely absorptive 2D electronic spectra recorded at room temperature. The color scale corresponds to $\Delta \mathrm{A} \times 10^{4}$ and the contour lines mark approximately $10 \%$ steps. Negative/positive amplitudes represent decay/rise of the bleaching.

a

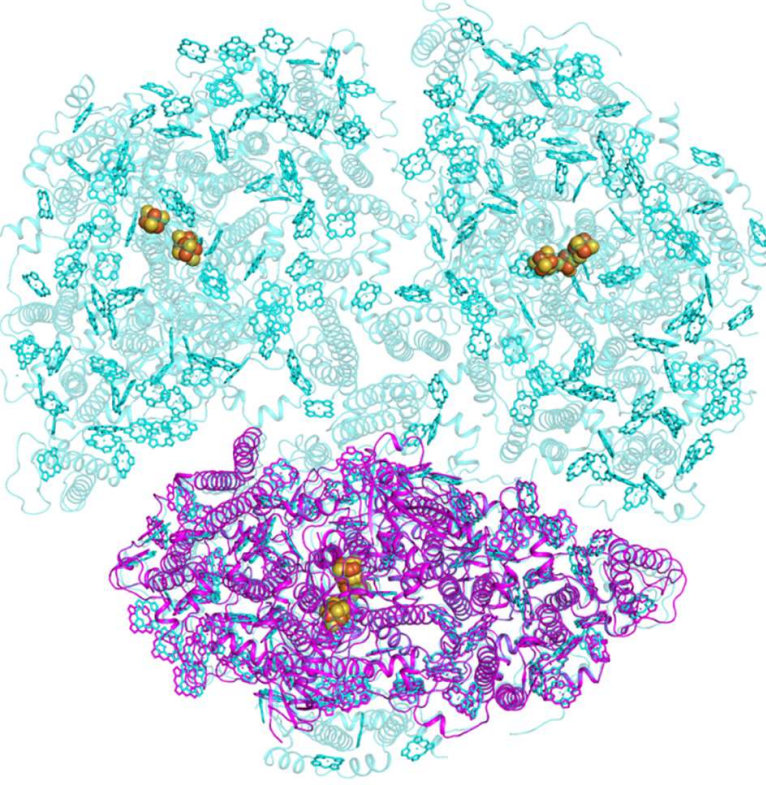

b

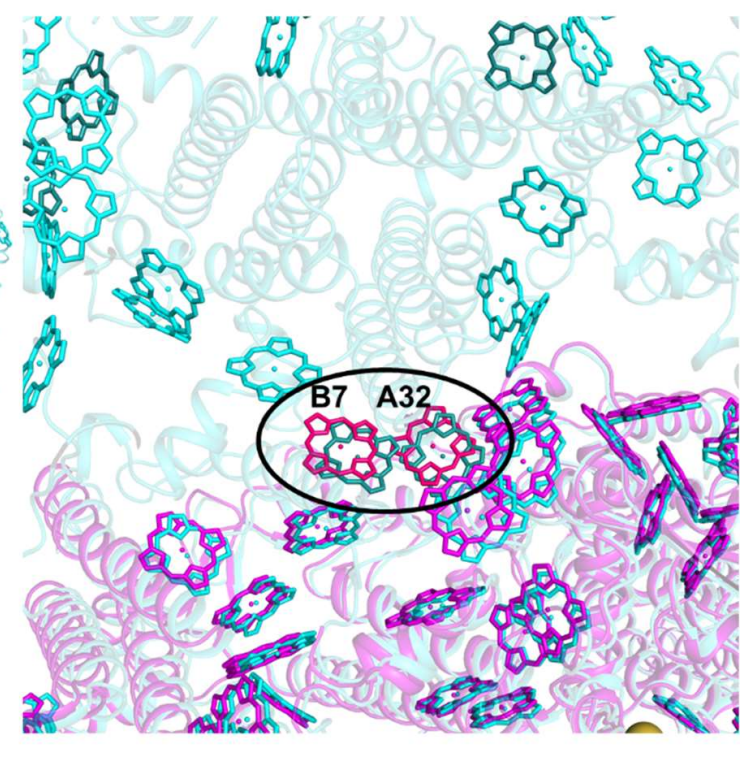

Figure S 8. Comparison of the structure of wild-type and $\Delta$ FIJL Photosystem I. a) overlay of the structure of trimeric PSI from Synechocystis PCC 6803 (PDB 50Y0, light blue) and $\Delta$ FIJL PSI (PDB 701V, purple). b) zoomin of the monomerization region highlighting the Chl pair A32-B7. 

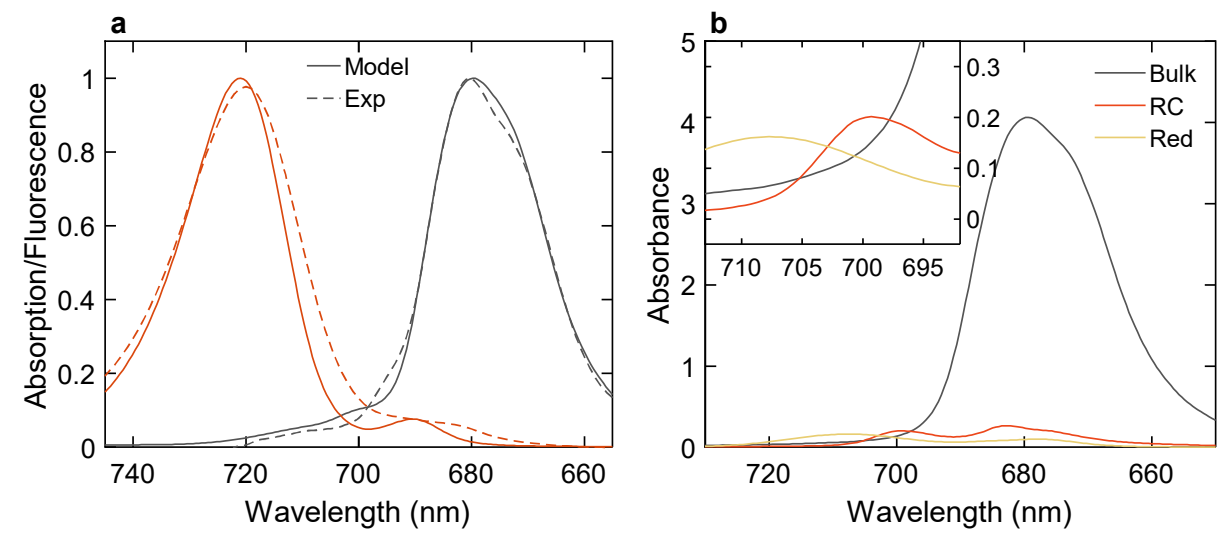

Figure S 9. Comparison of the structure-based model and experimental steady-state spectra. a) Model (solid lines) and experimental (dashed lines) absorption (black) and emission (spectra) spectra at $77 \mathrm{~K}$, normalized at the maximum. b) Model absorption spectra of the bulk antenna Chls, the six RC Chls and the two red Chl pairs B31-B32 and B37-B38.
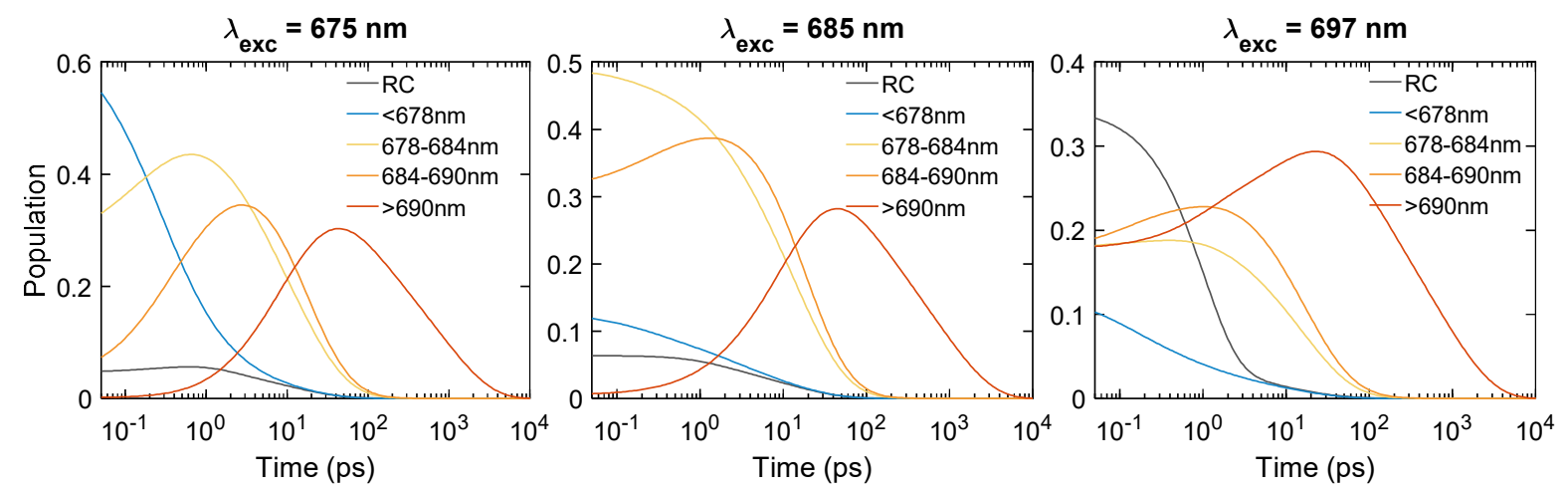

Figure S 10. Time-dependent aggregated exciton population calculated from the structure-based RedfieldFörster EET model for three excitation wavelengths $\lambda_{\text {exc. }}$ The curves represent the summed population of the six RC Chls and groups of antenna states with transition energies corresponding to wavelengths $<678 \mathrm{~nm}, 678$ $682 \mathrm{~nm}, 682-704 \mathrm{~nm}$ and $>704 \mathrm{~nm}$ (red Chls). 

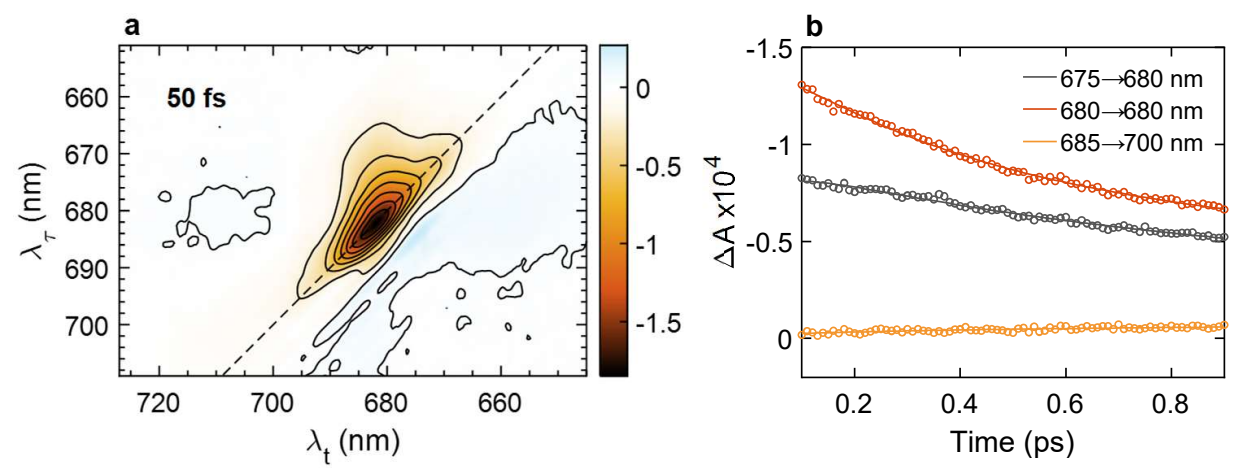

Figure S 11. 2DES data collected from PSI from Synechocystis $\Delta$ FIJL at $77 \mathrm{~K}$ for FT 2D frequency maps. (a) 2D electronic spectrum at 50 fs. (b) Kinetic traces recorded over a $T_{w}$ range of $0-1$ ps with a 10-fs step. In our present setup, the 2D electronic spectra are acquired automatically as absorptive, and cannot be trivially decomposed to the rephasing and non-rephasing signals. We perform Fourier transform of the absorptive 2D signal along waiting time $T_{w}$, after subtracting the exponential decay terms, resulting in 2D FT maps for beating frequencies $0-1600 \mathrm{~cm}^{-1}$ with $33 \mathrm{~cm}^{-1}$ resolution. Selected 2D FT maps at frequencies of $104 \mathrm{~cm}^{-1}$ and 243 $\mathrm{cm}^{-1}$ are presented in Figure 8. 


\section{SUPPLEMENTARY TABLES}

Table S1. Cryo-EM structure analysis parameters of $\Delta$ FIJL Photosystem I

$\triangle$ FIJL (EMDB-12697, PDB 701V)

\begin{tabular}{|c|c|}
\hline \multicolumn{2}{|l|}{$\begin{array}{l}\text { Data collection and } \\
\text { processing }\end{array}$} \\
\hline Magnification & 165,000 \\
\hline Voltage (kV) & 300 \\
\hline Electron exposure $\left(\mathrm{e}^{-} / \AA^{2}\right)$ & 43.485 \\
\hline Defocus range $(\mu \mathrm{m})$ & $0.9-3.0$ \\
\hline Pixel size $(\AA)$ & 0.8328 \\
\hline Symmetry imposed & C1 \\
\hline Initial particle images (no.) & 286,538 \\
\hline Final particle images (no.) & 74,303 \\
\hline Map resolution $(\AA)$ & 4.31 \\
\hline FSC threshold & 0.143 \\
\hline Map resolution range $(\AA)$ & $3.5-4.5$ \\
\hline \multicolumn{2}{|l|}{ Refinement } \\
\hline $\begin{array}{l}\text { Initial model used (PDB } \\
\text { code) }\end{array}$ & $50 Y 0$ \\
\hline Model resolution $(\AA)$ & 2.5 \\
\hline FSC threshold & NA \\
\hline Model resolution range $(\AA)$ & 2.5 \\
\hline Map sharpening B factor $\left(\AA^{2}\right)$ & -193.5 \\
\hline \multicolumn{2}{|c|}{ Model composition } \\
\hline Non-hydrogen atoms & 20914 \\
\hline Protein residues & 7 \\
\hline Ligands & 120 \\
\hline \multicolumn{2}{|l|}{ B factors $\left(\AA^{2}\right)$} \\
\hline Protein & $68.56-157.63$ \\
\hline Ligand & $49.65-184.16$ \\
\hline \multicolumn{2}{|l|}{ R.m.s. deviations } \\
\hline Bond lengths $(\AA)$ & 0.007 \\
\hline Bond angles $\left({ }^{\circ}\right)$ & 1.951 \\
\hline \multicolumn{2}{|l|}{ Validation } \\
\hline MolProbity score & 1.90 \\
\hline Clashscore & 10.07 \\
\hline Poor rotamers (\%) & 0.07 \\
\hline \multicolumn{2}{|l|}{ Ramachandran plot } \\
\hline Favored (\%) & 94.56 \\
\hline Allowed (\%) & 5.39 \\
\hline Disallowed (\%) & 0.05 \\
\hline
\end{tabular}


Table S2. Exciton domains in the minimal Photosystem I defined at cutoff energy $V_{c}=70 \mathrm{~cm}^{-1}$

\begin{tabular}{llll}
\hline No. & Chls & No. & Chls \\
\hline 1 & RC & 13 & A38-A39 \\
\hline 2 & A2-A3 & 14 & B1-B2 \\
\hline 3 & A6-A7 & 15 & B4-B5 \\
\hline 4 & A8-A10-A18 & 16 & B9-B17 \\
\hline 5 & A12-A14 & 17 & B14-B15-B23 \\
\hline 6 & A16-A17-A25 & 18 & B18-B19-B40 \\
\hline 7 & A20-A21-A22-PL1 & 19 & B20-B21 \\
\hline 8 & A24-A35-A37 & 20 & B22-B34-B36 \\
\hline 9 & A26-A27 & 21 & B24-B25 \\
\hline 10 & A29-A30 & 22 & B27-B28 \\
\hline 11 & A31-A32-B7 & 23 & B31-B32 \\
\hline 12 & A33-A34 & 24 & B37-B38 \\
\hline
\end{tabular}

Table S3. The strongest excitonic couplings in monomeric and trimeric Photosystem I complexes

\begin{tabular}{ccrrr}
\hline \multicolumn{2}{c}{ Chl pairs } & Minimal PSI & $\begin{array}{r}\text { Synechocystis } \\
\text { PCC }^{\mathbf{6 8 0 3}}{ }^{+}\end{array}$ & T. elongatus $^{\ddagger}$ \\
\hline ecA1 & ecB1 & 429 & 354 & 415 \\
\hline A12 & A14 & -211 & -235 & -231 \\
\hline ecB2 & ecB3 & 210 & 162 & 189 \\
\hline B31 & B32 & $-\mathbf{2 0 9}$ & $\mathbf{- 2 5 1}$ & $\mathbf{- 2 4 8}$ \\
\hline ecA2 & ecA3 & 187 & 136 & 157 \\
\hline B09 & B17 & -158 & -174 & -179 \\
\hline A33 & A34 & -153 & -155 & -162 \\
\hline B37 & B38 & $-\mathbf{1 5 0}$ & $\mathbf{- 1 8 7}$ & $-\mathbf{2 0 6}$ \\
\hline A10 & A18 & -136 & -193 & -181 \\
\hline A31 & A32 & 131 & 150 & 146 \\
\hline B35 & B36 & -127 & -120 & -121 \\
\hline B18 & B19 & -127 & -160 & -124 \\
\hline A26 & A27 & -120 & -147 & -138 \\
\hline B24 & B25 & -120 & -140 & -135 \\
\hline A32 & B07 & $\mathbf{1 1 9}$ & $\mathbf{1 7 6}$ & $\mathbf{2 1 1}$ \\
\hline A16 & A25 & -118 & -107 & -113 \\
\hline A29 & A30 & -118 & -114 & -111 \\
\hline A36 & A37 & -115 & -152 & -141 \\
\hline B14 & B23 & -112 & -90 & -97 \\
\hline A06 & A07 & -109 & -106 & -108 \\
\hline
\end{tabular}

† Calculated using the structure in ref. 1

‡ From ref. 2 
Table S4. Population decay lifetimes and amplitudes for the RC and select groups of antenna exciton states after excitation at $675 \mathrm{~nm}$

\begin{tabular}{lrrrrrr}
\hline Excitons & $\boldsymbol{\tau}_{\mathbf{1}}$ (ps) & $\boldsymbol{a}_{\mathbf{1}}$ & $\boldsymbol{\tau}_{\mathbf{2}}$ (ps) & $\boldsymbol{a}_{\mathbf{2}}$ & $\boldsymbol{\tau}_{\mathbf{3}}$ (ps) & $\boldsymbol{a}_{\mathbf{3}}$ \\
\hline $\mathrm{RC}$ & 0.3 & -0.02 & 3.3 & 0.03 & 23 & 0.03 \\
\hline$<678 \mathrm{~nm}$ & 0.2 & 0.34 & 1.1 & 0.24 & 13 & 0.07 \\
\hline $678-684 \mathrm{~nm}$ & 0.2 & -0.17 & 6.0 & 0.23 & 28 & 0.24 \\
\hline $685-690 \mathrm{~nm}$ & 0.2 & -0.13 & 1.0 & -0.17 & 24 & 0.32 \\
\hline$>700 \mathrm{~nm}$ & 9.0 & -0.30 & 260 & 0.15 & 1490 & 0.18 \\
\hline
\end{tabular}




\section{DYNAMIC THEORY OF OPTICAL SPECTRA}

\section{Exciton Hamiltonian}

The Frenkel exciton Hamiltonian of the Chl-protein complex has the form:

$$
H_{\mathrm{ex}}=\sum_{m} E_{m}|m\rangle\left\langle m\left|+\frac{1}{2} \sum_{n \neq m} V_{m n}\right| m\right\rangle\langle n|
$$

where $|m\rangle$ is a local excited state, in which the $m^{\text {th }} \mathrm{Chl}$ is in the first singlet excited state and all other Chls are in the ground state, $E_{m}$ is the unperturbed transition energy (site energy) and $V_{m n}$ is the excitonic (Coulomb) coupling between Chls $m$ and $n$.

The exciton coupling is calculated adopting dipole approximation:

$$
V_{m n}=5.04 C\left(\frac{\vec{\mu}_{m} \cdot \vec{\mu}_{n}}{R^{3}}-\frac{3\left(\overrightarrow{\mathrm{r}}_{m n} \cdot \vec{\mu}_{m}\right)\left(\overrightarrow{\mathrm{r}}_{m n} \cdot \vec{\mu}_{n}\right)}{R^{5}}\right)
$$

where $\vec{\mu}_{m}$ is the $m^{\text {th }}$ site transition dipole moment given in unit of Debye, $\overrightarrow{\mathrm{r}}_{m n}$ and $R$ are the centerto-center vector connecting the Chls and the distance, in $\mathrm{nm}$. For the present calculations, the value of the dipole strength of Chl $a$ was set to $21 \mathrm{D}^{2}$. The constant $C$ reflects the environment, and is calculated from the refractive index $(n=1.4)$ as:

$$
C=\frac{\left(n^{2}+2\right)^{2}}{9 n^{2}}
$$

Delocalized exciton states are obtained by diagonalizing the Hamiltonian:

$$
|\alpha\rangle=\sum_{m} c_{m}^{\alpha}|m\rangle
$$

The excitonic Hamiltonian of the entire complex is partitioned into exciton domains consisting of strongly coupled Chls, wherein $V_{m n}>V_{\mathrm{c}}$, with the implicit assumption that the exciton coupling is stronger than the reorganization energy of the Chl-protein coupling. In the present calculations we have chosen $V_{\mathrm{c}}=70 \mathrm{~cm}^{-1}$, which places all six Chls in the PSI RC in the same exciton domain and all other exciton domains containing no more than three Chls, with the exception of the quartet A20A21-A22-PL1.

\section{Absorption and emission lineshapes}

A dynamic theory of optical spectra was applied to calculate the absorption and emission lineshapes of the excitonic states and the aggregate spectra ${ }^{3}$. The lineshape function is obtained from POPtheory within secular approximation and Markov approximation for the off-diagonal elements of the exciton-vibrational coupling. The exciton absorption and emission lineshapes are given by

$$
D_{\alpha}(\omega)=\frac{1}{2 \pi} \int_{-\infty}^{\infty} \mathrm{d} t e^{i\left(\omega-\widetilde{\omega}_{\alpha}\right) t} e^{G_{\alpha}(t)-G_{\alpha}(0)} e^{-\Gamma_{\alpha}|t|}
$$




$$
D_{\alpha}^{\prime}(\omega)=\frac{1}{2 \pi} \int_{-\infty}^{\infty} \mathrm{d} t e^{-i\left(\omega-\widetilde{\omega}_{\alpha}\right) t} e^{G_{\alpha}(t)-G_{\alpha}(0)} e^{-\Gamma_{\alpha}|t|}
$$

where $G_{\alpha}(t)$ describes the vibrational side band of the transition, $\widetilde{\omega}_{\alpha}$ is the (renormalized) exciton transition frequency ${ }^{3}$ and the last term represents lifetime broadening with the dephasing rate

$$
\Gamma_{\alpha}=\frac{1}{2} \sum_{\beta}^{\beta \neq \alpha} k_{\alpha \rightarrow \beta}
$$

where $k_{\alpha \rightarrow \beta}$ is a Redfield relaxation rate (see below).

The time-dependent function $G_{\alpha}(t)$ is

$$
G_{\alpha}(t)=\gamma_{\alpha \alpha} \int_{0}^{\infty} \mathrm{d} \omega\left((1+n(\omega)) J(\omega) e^{-i \omega t}+n(\omega) J(\omega) e^{i \omega t}\right)
$$

Here $n(\omega)$ is a Bose-Einstein factor

$$
n(\omega)=\frac{1}{e^{\hbar \omega / k_{B} T}-1}
$$

and $J(\omega)$ is the spectral density of coupling to protein vibrations $U(\omega)=0$ for $\omega<0)$ :

$$
J(\omega)=\sum_{\xi} g_{\xi}^{2} \delta\left(\omega-\omega_{\xi}\right)
$$

with $g_{\xi}$ giving the coupling to a vibrational mode with frequency $\omega_{\xi}$.

The spectral density is related to the Huang-Rhys factor $S$ and the reorganization energy $\lambda$ :

$$
\begin{gathered}
S=\int_{0}^{\infty} \mathrm{d} \omega J(\omega) \\
\lambda=\hbar \int_{0}^{\infty} \omega \mathrm{d} \omega J(\omega)
\end{gathered}
$$

In the present calculations, high-frequency intramolecular vibrations are neglected and the spectral density is described analytically as a continuous function 4 :

$$
J(\omega)=\frac{1}{s_{1}+s_{2}} \sum_{i=1}^{2} \frac{s_{i}}{7 ! 2 \varepsilon_{i}^{4} / \hbar^{4}} \omega^{3} e^{-\sqrt{\hbar \omega / \varepsilon_{i}}}
$$

with parameters $s_{1}=0.8, \varepsilon_{1}=0.56 \mathrm{~cm}^{-1}, s_{2}=0.5, \varepsilon_{2}=1.94 \mathrm{~cm}^{-1}$.

The coefficient $\gamma_{\alpha \alpha}$ is the diagonal part of

$$
\gamma_{\alpha \beta}=\sum_{m, n} c_{m}^{\alpha} c_{n}^{\alpha} c_{m}^{\beta} c_{n}^{\beta} e^{-R_{m n} / R_{c}}
$$


where $c_{m}^{\alpha}$ are exciton coefficients, $R_{m n}$ is the center-to-center distance between Chls $m$ and $n$ and $R_{c}$ is the correlation radius of protein vibrations; we use $R_{c}=5 \AA^{4}$.

The $0-0$ transition frequency is renormalized by the exciton-vibrational coupling:

$$
\widetilde{\omega}_{\alpha}=\omega_{\alpha}-\gamma_{\alpha \alpha} \frac{\lambda}{\hbar}+\sum_{\beta \neq \alpha} \gamma_{\alpha \beta} \text { P.V. } \int_{-\infty}^{\infty} \mathrm{d} \omega \frac{\omega^{2}((1+n(\omega)) J(\omega)+n(-\omega) J(-\omega))}{\omega_{\alpha \beta}-\omega}
$$

Here $\omega_{\alpha \beta}$ is the transition frequency difference between exciton states, $\lambda$ is the reorganization energy, P.V. denotes principal value of the integral, and $J(\omega)=0$ for $\omega<0$.

\section{Exciton dynamics}

The exciton relaxation dynamics within strongly coupled exciton domains is calculated using modified Redfield theory e.g. ${ }^{3}$. Exciton relaxation between states $|\alpha\rangle$ and $|\beta\rangle$, belonging to the same exciton domain, is described by the Redfield rate constant

$$
k_{\alpha \rightarrow \beta}=2 \pi \gamma_{\alpha \beta} \omega_{\alpha \beta}^{2}\left(\left(1+n\left(\omega_{\alpha \beta}\right)\right) J\left(\omega_{\alpha \beta}\right)+n\left(\omega_{\beta \alpha}\right) J\left(\omega_{\beta \alpha}\right)\right)
$$

The rates of exciton transfer between excited states belonging to different domains are calculated (in $\mathrm{ps}^{-1}$ ) using generalized Förster theory ${ }^{5-7}$ :

$$
k_{\alpha_{k} \rightarrow \beta_{l}}=1.183 \times\left|V_{\alpha_{k} \beta_{l}}\right|^{2} \int_{-\infty}^{\infty} \mathrm{d} \omega D_{\alpha_{k}}^{\prime}(\omega) D_{\beta_{l}}(\omega)
$$

The exciton lineshapes are area-normalized. The interdomain exciton coupling (in $\mathrm{cm}^{-1}$ ) is calculated from the monomer coupling energies as:

$$
V_{\alpha_{k} \beta_{l}}=\sum_{m_{k}, n_{l}} c_{m_{k}}^{\alpha_{k}} c_{n_{k}}^{\beta_{k}} V_{m_{k} n_{k}}
$$

The exciton population dynamics $\mathbf{p}(t)$ is calculated for different initial excitation conditions using the master equation:

$$
\frac{\mathrm{d} \mathbf{p}}{\mathrm{d} t}=\mathbf{K p}(t)
$$

The off-diagonal elements of the transfer matrix are the rate constants of exciton transfer and the diagonal elements are $k_{m m}=-\sum_{n} k_{m \rightarrow n}-k_{\mathrm{d}}$, with the additional deactivation rate $k_{\mathrm{d}}$ equal to 1.5 $\mathrm{ps}^{-1}$ for the lowest-energy state of the RC and $0.5 \mathrm{~ns}^{-1}$ for all other states. The master equation is solved by matrix decomposition yielding eigenvalues $\boldsymbol{\Lambda}$ (inverse population decay lifetimes) and right eigenvectors $\mathbf{U}$.

\section{Stationary and time-dependent optical spectra}

The homogeneous excitonic absorption and emission spectra are given by

$$
\begin{aligned}
& a_{\alpha}^{\text {hom }}=\left|\mu_{\alpha}\right|^{2} D_{\alpha}(\omega) \\
& f_{\alpha}^{\text {hom }}=\left|\mu_{\alpha}\right|^{2} D_{\alpha}^{\prime}(\omega)
\end{aligned}
$$


where the $\mu_{\alpha}$ is the excitonic transition dipole moment. The steady-state absorption spectrum of the complex is

$$
A(\omega)=\left\langle\sum_{\alpha} a_{\alpha}^{\text {hom }}\right\rangle
$$

where $\langle\ldots\rangle$ indicates disorder averaging. Only diagonal disorder (in site energies) was considered and Monte Carlo sampling was performed in the usual way.

The stationary fluorescence emission spectrum is calculated by integrating the time-dependent emission:

$$
F(\omega)=\left\langle\sum_{\alpha} f_{\alpha}^{\mathrm{hom}}(\omega) \int_{0}^{\infty} \mathrm{d} t p_{\alpha}(t)\right\rangle
$$

where $p_{\alpha}(t)$ is the time-dependent population of state $|\alpha\rangle$.

Finally, for a qualitative comparison with the time-resolved spectroscopy data, time-dependent bleaching / stimulated emission spectra are simulated as

$$
\Delta A(\omega ; t)=-\left\langle\sum_{\alpha}\left(a_{\alpha}^{\text {hom }}(\omega)+f_{\alpha}^{\text {hom }}(\omega)\right) p_{\alpha}(t)\right\rangle
$$

Note that photoinduced absorption (of excited-states or charge-separated states) as well as coherence dynamics are not simulated.

\section{REFERENCES}

1. Malavath, T.; Caspy, I.; Netzer-El, S. Y.; Klaiman, D.; Nelson, N., Structure and function of wild-type and subunit-depleted photosystem I in Synechocystis. Biochim. Biophys. Acta 2018, 1859 (9), 645654.

2. Byrdin, M.; Jordan, P.; Krauss, N.; Fromme, P.; Stehlik, D.; Schlodder, E., Light harvesting in photosystem I: modeling based on the 2.5-Å structure of photosystem I from Synechococcus elongatus. Biophys. J. 2002, 83 (1), 433-457.

3. Raszewski, G.; Renger, T., Light Harvesting in Photosystem II Core Complexes Is Limited by the Transfer to the Trap: Can the Core Complex Turn into a Photoprotective Mode? J. Am. Chem. Soc. 2008, 130 (13), 4431-4446.

4. Renger, T.; Marcus, R., On the relation of protein dynamics and exciton relaxation in pigmentprotein complexes: an estimation of the spectral density and a theory for the calculation of optical spectra. J. Chem. Phys. 2002, 116 (22), 9997-10019.

5. Sumi, H., Theory on rates of excitation-energy transfer between molecular aggregates through distributed transition dipoles with application to the antenna system in bacterial photosynthesis. J. Phys. Chem. B 1999, 103 (1), 252-260.

6. Scholes, G. D.; Fleming, G. R., On the mechanism of light harvesting in photosynthetic purple bacteria: B800 to B850 energy transfer. J. Phys. Chem. B 2000, 104 (8), 1854-1868.

7. Jang, S.; Newton, M. D.; Silbey, R. J., Multichromophoric Förster resonance energy transfer. Phys. Rev. Lett. 2004, 92 (21), 218301. 